\title{
Fermion Chern Simons Theory of Hierarchical Fractional Quantum Hall States
}

\author{
Ana López ${ }^{1}$, and Eduardo Fradkin ${ }^{2}$ \\ ${ }^{1}$ Department of Physics, Theoretical Physics, Oxford University, 1 Kebble Road, Oxford OX2 3NP, UK \\ ${ }^{2}$ Department of Physics, University of Illinois at Urbana-Champaign, \\ 1110 W. Green St., Urbana IL 61801-3080, USA
}

(Dated: November 7, 2018)

\begin{abstract}
We present an effective Chern-Simons theory for the bulk fully polarized fractional quantum Hall (FQH) hierarchical states constructed as daughters of general states of the Jain series, $i$. e. as FQH states of the quasi-particles or quasi-holes of Jain states. We discuss the stability of these new states and present two reasonable stability criteria. We discuss the theory of their edge states which follows naturally from this bulk theory. We construct the operators that create elementary excitations, and discuss the scaling behavior of the tunneling conductance in different situations. Under the assumption that the edge states of these fully polarized hierarchical states are unreconstructed and unresolved, we find that the differential conductance $G$ for tunneling of electrons from a Fermi liquid into any hierarchical Jain FQH states has the scaling behavior $G \sim V^{\alpha}$ with the universal exponent $\alpha=1 / \nu$, where $\nu$ is the filling fraction of the hierarchical state. Finally, we explore alternative ways of constructing FQH states with the same filling fractions as partially polarized states, and conclude that this is not possible within our approach.
\end{abstract}

In 1983 Laughlin [1] proposed his celebrated wave functions as an explanation of the fractional quantum Hall effect (FQHE) for a two-dimensional electron gas (2DEG) with filling factors $\nu=1 / m$, with $m$ an odd integer. Shortly after that, hierarchical generalizations of these fully polarized states were also proposed for arbitrary filling factors with odd-denominator fractions [2 3]. The basic idea behind these Halperin-Haldane hierarchical states is that, at filling factors away from $\nu=1 / m$, there is a finite density of excitations of a primary (Laughlin) fractional quantum Hall $(\mathrm{FQH})$ state, that are quasiholes with fractional charge and fractional statistics, which themselves may also condense into a new incompressible FQH state. Thus, for instance the FQH state at $\nu=2 / 5$ is viewed as a FQH state of quasi-holes of the $\nu=1 / 3$ state, a "condensate". Away from these filling factors, there is a finite density of these new excitations which in turn may also condense into yet another incompressible FQH state, and so on. The bosonic ChernSimons field theory of the Laughlin states [4] describes the FQH states as the Bose condensation of a (bosonic) field. In this picture the Haldane-Halperin hierarchical states are described by the condensation of vortex excitations on the primary condensate. [5]

Thus, Halperin-Haldane hierarchical FQH states have a built-in nested structure, much reminiscent to that of a Russian doll, in which states higher in the hierarchy are supported by lower states and so forth. Implicit in this construction is the assumption that, as the magnetic field or the density are varied, their excitations remain well defined even when their densities are relatively high (as measured by their effective filling factors). Besides, underlying lower states remain stable and inert as their excitations condense into yet new higher states. Thus, although the Halperin-Haldane hierarchy offers a simple way to construct states for arbitrary filling, it is a crude model whose assumptions may not obviously hold. Furthermore, it is a necessary consequence of the assump- tions of this hierarchical construction that the Hilbert spaces supported by these states have many conserved currents, one for each level of the hierarchy, even though the underlying 2DEG a priori has only one conserved current, the charge current, as required by charge conservation. Thus even if the Halperin-Haldane hierarchical states may be sound in the low energy limit, at the microscopic level most of these additional conservation laws must be violated. Hence, for this hierarchy to work it is necessary to suppress the processes which violate the extra conservation laws. It also follows from these constructions that, as the physical edges of the 2DEG are approached, the intricate structures of these states is progressively revealed as one layer after another of the state structure gets peeled-off [ $[6]$. This nested structure also determines many qualitative features of the phase diagram of the 2DEG in the regime in which only incompressible fluids and insulating states are present 7 ].

An alternative construction, based on the notion of composite fermions, was proposed by Jain [8]. This approach yields stable $\mathrm{FQH}$ states for filling factors on the sequences $1 / \nu=2 n+1 / p$, where $n$ and $p$ are integers $(n \geq 0)$. The FQH states on the Jain sequences are the most prominent, and hence the most stable, of the experimentally observed FQH states. In these families of states, within a mean-field picture, the composite fermions fill up a finite number $|p|$ of Landau levels of a partially screened magnetic field. Beyond mean field theory fluctuation effects, embodied by an effective Chern-Simons gauge field [9], turn the mean field composite fermions into excitations with fractional charge and fractional statistics [9]. It is also possible to construct a hierarchy of FQH states using composite fermions [10 11].

Although the universal physical properties of the FQH states, i.e. the charge and statistics of the excitations and the ground state degeneracy, derived from both constructions are in fact identical [4, 6 9 , in practice the nature of the approximations that are made are different in each 
approach, $i$. e. condensation of a composite boson or filling up Landau levels of composite fermions. As a result, there are significant quantitative differences in their predictions of energy gaps and other non-universal but physically important properties. Besides, as we already discussed, it is an experimental fact that the states in the main Jain sequence are the most stable fully polarized states, while within the Haldane-Halperin approach, only the Laughlin states would be the naturally more stable. Why this is true is not entirely clear theoretically since these theories (bosonic or fermionic) do not have a small expansion parameter and the corrections to the mean field results may be large (and in practice are large). Typically mean field theory overestimates the energy gaps of the Jain states by about an order of magnitude (compared with numerical results for small systems). Also, the flux attachment transformation, central to both constructions, is a local operation in space and as such it involves a large amount of Landau level mixing. While this is not a problem for the determination of the universal data of FQH states, it does have a large effect on energy gaps and similar quantities. In particular the mean field theory yields gaps whose magnitude is controlled by the effective magnetic field acting on the composite fermions and not by the physical energy scale, the Coulomb energy at the magnetic length. Calculations beyond mean field theory may solve in the end some of these problems 121314 . Numerical calculations with a relatively small number of electrons, presumably with significant and yet poorly understood finite size effects, support the conclusion that the Jain states are indeed more stable and that their stability diminishes with the order of the Jain sequence 15. Thus, at least qualitatively, the stability of the experimentally observed states, as measured by the width of their respective Hall conductivity plateaus, follows the progression of the Jain sequences, rather than the Haldane-Halperin hierarchy [2]3].

As we noted above, the Halperin-Haldane hierarchy leads to an effective low-energy theory which involves a number of conserved currents, one per level of the hierarchy, which thus grows with the level. This is actually a feature inherent to all hierarchical constructions, either fermionic or bosonic. In the $K$-matrix form of the effective theory, this feature is encoded in the rank of the $K$ matrix and on the number of fundamental quasiparticles, both of which grow with the level of the hierarchy [56]. It was noted by Haldane [16] that in most cases the resulting $K$-matrix has "null vectors", representing neutral particles with "zero statistics" (i.e. without chirality), and proposed that such particles need not be conserved thus leading to an instability of these states. He then went on to propose that the states whose $K$-matrices do not have null vectors are the only stable states and coined the term $T$-stability to describe this criterion.

Motivated by the question of whether these additional conservation laws are actually needed to understand the FQH states we have recently constructed a theory for all FQH states in the Jain sequences which requires the existence of only one conserved current, the charge current, and only one fundamental quasiparticle 17 18. A key ingredient of this theory is a generalization of the flux attachment transformation which is consistent with the requirements resulting from topological and gauge invariance applicable for a 2DEG on a closed surface. Unlike the standard hierarchy, the resulting effective ChernSimons theory has several components and it is characterized by a $K$-matrix whose rank is the same for all the sequences. Moreover the resulting theory is automatically $T$-stable in the sense of Haldane.

Recently, Pan et al. [19] have carried out a set of experiments on high mobility samples at very low temperatures in which they observed the FQHE in the lowest Landau level at filling fractions not included in the quantum Hall (Jain) series. A deep minimum in $\rho_{x x}$, as well as a respectable plateau in $\rho_{x y}$, was observed for $\nu=4 / 11$ and $5 / 13$, whereas for $\nu=6 / 17,4 / 13,5 / 17$, and $7 / 11$, the minimum was not as pronounced, and in fact no plateau in $\rho_{x y}$ was actually observed for these filling fractions. The state at $7 / 11$ was observed earlier by Goldman and Shayegan [20]. A respectable minimum in $\rho_{x x}$ was also seen for $\nu=3 / 8$, but without any evidence for a plateau in $\rho_{x y}$. In the case of the best defined of these new states, with $\nu=4 / 11$, Pan et al. [19] suggested that it is a fully polarized FQH state. They further proposed that the states they observed are evidence of a FQHE of composite fermions.

If this interpretation is correct, these are the first truly hierarchical FQH states observed to date, i. e. FQH states of the physical excitations of a primary Jain state. However, we should note that there are other possible mechanisms to get an incompressible FQH fluid, at least for some of the filling fractions they observed. Finite size diagonalization of small clusters of electrons 2122.23] suggest that some of the observed states, such as the one at $\nu=4 / 11$, should be at most partially polarized. This result is, however, at variance with the experiments of Ref. [19] that are consistent with a fully polarized state. On the other hand, a number of authors 24 25.26] have also proposed that at least for some of the observed fractions, for example $\nu=3 / 8$, the ground state may be a paired quantum Hall state, with pairing of the excitations of the Laughlin $\nu=1 / 3 \mathrm{FQH}$ state [27]. It is clear that in principle there may be two or more competing phases and that whichever state is observed may depend on subtle microscopic details.

In this paper we will take the point of view that the observed states are indeed hierarchical Jain states. In other words, these new states are the result of the condensation of the physical quasi-particles and quasi-holes of the primary Jain states into new Jain-like FQH states. We will use here the approach we introduced in Refs. [17, 18] to construct these new states as hierarchical Jain states, and to derive an effective Chern-Simons field theory for these states, demanding the existence of only the minimum number of necessary conservation laws and compatible with the consistency requirements when the 2DEG is 
placed on a closed surface. We will show that these states can be (locally) stable and that they satisfy the requirements for $T$-stability. We will also show that these new states are organized more naturally as Jain hierarchical states rather than along the lines suggested by the standard Haldane-Halperin hierarchy. We will use the resulting bulk theory to find an effective theory of an unreconstructed unresolved edge and to compute the tunneling exponent for electrons into these states. We find that it is equal to $1 / \nu$ for all systems with unreconstructed, unresolved ( $i$. e. "sharp") edge states within the Jain series as well as for all of their hierarchical descendants. This result, which we found earlier to hold for the primary Jain states [17], is consistent with all the presently available experimental data on the tunneling exponent 282930 31]. It suggests that this dependence of the exponent on the filling factor is a generic property of all clean unreconstructed unresolved edges [32]. In contrast, it is well known that if the edges are resolved, whether they are clean or not, the exponent has a more complex dependence on the filling fraction $33,34,35,36$.

The paper is organized as follows. In Section \we introduce a generalization of the fermionic Chern-Simons theory of the FQHE for fully polarized systems along the lines of Refs. [9 17], and use it to construct these states, to compute their degeneracy on a torus, and to determine the quantum numbers of their quasi-hole and quasi-particle excitations. We also show that, contrary to the general expectations derived from the standard hierarchy [16] these states are $T$-stable. In doing so we will assume that quasi-particles and quasi-holes of the primary Jain states have simple and short range interactions. Under these assumptions it is possible to give a simple (perhaps naive) criterion for the stability of these states, which is discussed in Section III In Section III we study the possibility of these states being realized as partially polarized primary FQH state of electrons, and conclude that this option is not generally feasible within our approach, for all the filling fractions that have been observed. Finally in Section IV we derive an effective theory for the edge states for these new hierarchical states, and calculate the electron edge tunneling exponents.

\section{FULLY POLARIZED HIERARCHICAL JAIN STATES}

The elementary excitations of all FQH states, including those on the principal Jain sequences with filling factor $1 / \nu=2 n+1 / p$, are quasi-holes with fractional charge and fractional statistics [1 2, 4 , 5 , 9]. Here we will take as the starting point, the effective theory for the states in the Jain sequences developed in our earlier work [17], which follows the framework and notation of Wen [5]. In this work the elementary excitations of these states are described by a set of currents $j_{q p}^{\mu}$, the world-lines of a set of (composite) fermions, which are coupled to a statistical gauge field $a_{\mu}$ and to a hydrodynamic gauge field $b_{\mu}$ through the effective action

$$
\begin{aligned}
\mathcal{L} & =\frac{p}{4 \pi} \epsilon_{\mu \nu \lambda} a_{\mu} \partial_{\nu} a_{\lambda}+\frac{1}{2 \pi} \epsilon_{\mu \nu \lambda} a_{\mu} \partial_{\nu} b_{\lambda} \\
& -\frac{2 n}{4 \pi} \epsilon_{\mu \nu \lambda} b_{\mu} \partial_{\nu} b_{\lambda}+\frac{1}{2 \pi} \epsilon_{\mu \nu \lambda} b_{\mu} \partial_{\nu} A_{\lambda}+a_{\mu} j_{q p}^{\mu}
\end{aligned}
$$

where $A_{\mu}$ is an external electromagnetic perturbation. This effective action reproduces all the universal data of the Jain states: the $2 n p+1$-fold ground state degeneracy on the torus, the quasi-particle fractional charge $e /(2 n p+1)$ and fractional statistics $2 n \pi /(2 n p+1)$ (measured relative to fermions) [17].

Away from these precise filling factors, the system has a finite density of quasi-particles (or quasi-holes) which, due to their residual interactions, can condense in a new FQH state in the field $a_{\mu}$. Thus, we will assume that the underlying Jain state remains stable even in the presence of a finite density of its elementary excitations. We can then apply the same procedure for these quasi-particles as we did for the original electrons, and attach an even number $2 n_{1}$ of flux quanta to each quasi-particle which ensures that their statistics remains unchanged, resulting in the quasi-particle Lagrangian:

$$
\begin{aligned}
\mathcal{L}_{q p} & =a_{\mu} j_{q p}^{\mu} \rightarrow a_{\mu} j_{q p}^{\mu}+c_{\mu} j_{q p}^{\mu}+\frac{1}{2 \pi} \epsilon_{\mu \nu \lambda} c_{\mu} \partial_{\nu} d_{\lambda} \\
& -\frac{2 n_{1}}{4 \pi} \epsilon_{\mu \nu \lambda} d_{\mu} \partial_{\nu} d_{\lambda}
\end{aligned}
$$

where we have written the conserved quasi-particle current as $j_{q p}^{\mu}=\frac{1}{2 \pi} \epsilon_{\mu \nu \lambda} \partial_{\nu} d_{\lambda}$. Upon integrating out the quasi-particles, within a mean field theory in which they fill up $\left|p_{1}\right|$ Landau levels of the effective field $a_{\mu}+\left\langle c_{\mu}\right\rangle$, the effective action becomes

$$
\begin{aligned}
\mathcal{L}_{q p} & =\frac{p_{1}}{4 \pi} \epsilon_{\mu \nu \lambda} c_{\mu} \partial_{\nu} c_{\lambda}+\frac{1}{2 \pi} \epsilon_{\mu \nu \lambda}\left(c_{\mu}+a_{\mu}\right) \partial_{\nu} d_{\lambda} \\
& -\frac{2 n_{1}}{4 \pi} \epsilon_{\mu \nu \lambda} d_{\mu} \partial_{\nu} d_{\lambda}
\end{aligned}
$$

where $c_{\mu}$ are the fluctuations about the mean field $\left\langle c_{\mu}\right\rangle$. Integrating out $c_{\mu}$ and $d_{\mu}$ we find that the contribution of the quasi-particles to the total action is $\frac{\nu_{1}}{4 \pi} \epsilon_{\mu \nu \lambda} a_{\mu} \partial_{\nu} a_{\lambda}$, with

$$
\frac{1}{\nu_{1}}=2 n_{1}+\frac{1}{p_{1}}
$$

i.e. the quasi-particles condense in a Jain-like FQH state with filling fraction $\nu_{1}$. So far, we have used the term quasi-particle to name the elementary excitations of the Jain states, independently of the sign of their charge. Notice, however, that if we take into account this sign, inter-changing quasi-particles (negative charge) by quasiholes (positive charge) will only change the sign of $\nu_{1}$. Thus, $\nu_{1}<0$ corresponds to a FQH state of quasi-holes and $\nu_{1}>0$ is a FQH state of quasi-particles (or quasielectrons). Hence there is no restriction on the sign of either $n_{1}$ or $p_{1}$. In contrast, we still have the constraint $n>0$ and that the total filling factor is positive. 
We can now collect the results of Eqs.11.1)-(1.3) in the more compact $K$-matrix form [5]. In this representation, the effective Lagrangian for a sequence of states whose "primary" states are the Jain ones at $1 / \nu=2 n+1 / p$ is :

$$
\mathcal{L}=\frac{1}{4 \pi} K_{I J} \epsilon_{\mu \nu \lambda} a_{\mu}^{I} \partial_{\nu} a_{\lambda}^{J}+\frac{1}{2 \pi} t_{I} \epsilon_{\mu \nu \lambda} a_{\mu}^{I} \partial_{\nu} A_{\lambda}+\ell_{I} a_{\mu}^{I} j_{q p, 1}^{\mu}
$$

where the coupling constant matrix is

$$
K=\left(\begin{array}{cccc}
-2 n & 1 & 0 & 0 \\
1 & p & 1 & 0 \\
0 & 1 & -2 n_{1} & 1 \\
0 & 0 & 1 & p_{1}
\end{array}\right)
$$

and the indices $I, J=1, . ., 4$. We have defined the gauge fields $a_{\mu}^{1}=b_{\mu}, a_{\mu}^{2}=a_{\mu}, a_{\mu}^{3}=d_{\mu}, a_{\mu}^{4}=c_{\mu}$, the charge vector $t=(1,0,0,0)$, and the flux vector $\ell=(0,0,0,1)$. $j_{q p, 1}^{\mu}$ is the quasi-particle current corresponding to the excitations above the hierarchical state. Therefore, the general set of allowed quasiparticle excitations on top of this hierarchical state can be represented by a vector $m=k \ell=(0,0,0, k)$ where $k \in \mathbb{Z}$.

It follows that the filling fraction of these states is $[5]$

$$
\nu=\left|t^{T} K^{-1} t\right|=\frac{p\left(2 n_{1} p_{1}+1\right)+p_{1}}{\operatorname{det} K}
$$

or, equivalently

$$
\frac{1}{\nu}=2 n+\frac{1}{p+\nu_{1}}
$$

where $\nu_{1}$ is given in Eq.(1.4). It is straightforward to check that these sequences include the series of FQH hierarchical states proposed earlier on in Ref. [10].

In Eq. (1.6), the quantity

$$
|\operatorname{det} K|=\left|\left(2 n_{1} p_{1}+1\right)(2 n p+1)+2 n p_{1}\right|
$$

is the ground state degeneracy of these states on a torus. This result agrees with the standard hierarchy even though, as we shall see, the spectrum is not quite the same. It is also easy to check that, provided $|\operatorname{det} K|$ remains finite, the excitation spectrum has a finite energy gap $E_{G}$ which in mean field theory is

$$
E_{G}=\frac{\hbar \omega_{c}}{|\operatorname{det} K|}
$$

We expect that this mean field result is an over-estimate of the size of the real gap. As it is usual in these type of approximations [8 9, 37] the mean field gap is just the cyclotron gap of the composite fermions and it depends on the bare mass (in this case of the quasi-particles or quasiholes which are condensing in the FQH state). Here too, on physical grounds one expects that the cyclotron scale should be replaced by the appropriate Coulomb interaction energy of two quasi-particles (or quasi-holes) at a distance of the order of the magnetic length. Thus, due to the short-distance structure of these excitations [38], we expect that quasi-particle and quasi-hole gaps will be appreciably reduced from the mean-field theory prediction.

The quantum numbers of the quasi-holes of the new states are

$$
Q_{q h}=e t^{T} K^{-1} \ell=\frac{e}{\operatorname{det} K}
$$

which is their charge, and

$$
\frac{\theta_{q h}}{\pi}=\ell^{T} K^{-1} \ell=\frac{2 n+2 n_{1}(2 n p+1)}{\operatorname{det} K}
$$

for their fractional statistics (measured from fermions). Note that there is only one quasi-particle (or quasi-hole) in our construction. In contrast, the Haldane-Halperin hierarchy predicts the existence of a number of well defined distinct stable quasi-particles whose number given by the rank of the matrix $K$, depends on the level of the hierarchy [5]. In particular, and in contrast to the requirements of the Haldane-Halperin hierarchical construction, in our theory there is one and only one conserved current with respect to the electromagnetic field.

An interesting consequence of this fact is that, in contrast with what happens in the case of the HaldaneHalperin hierarchical states, all the states described by our approach are $T$-stable. According to Haldane [16], an Abelian Quantum Hall theory is $T$-unstable if the effective $K$-matrix theory has quasiparticles labeled by vectors $m$ that satisfy $m K^{-1} m=0$ and $t K^{-1} m=0$. An important caveat is that the null vectors must belong to the subspace spanned by the $\ell$ vectors of the fundamental quasiparticles. In the standard hierarchy, the number of fundamental quasiparticles grows with the level of the hierarchy, i.e. with the rank of the $K$-matrix, and the null vectors (if they exist) belong to the physical subspace. In contrast, in our approach, the physical subspace is spanned by the fundamental quasiparticle, whose $\ell$-vector is $\ell=(0,0,0,1)$. Thus, although in all the cases we have discussed the $K$-matrices of the form of Eq. 1.6 have null vectors, they do not belong to the physical subspace. In other terms, in this construction the "null-particles" are unphysical, and as a result these FQH states are $T$-stable. In particular, the observed state at $\nu=4 / 11$ is $T$-stable [39].

Finally we note that this hierarchical construction, just as in any hierarchy, at least at a formal level can be repeated an indefinite number of times, leading to the construction of a (hierarchical) FQH Jain state for any odd-denominator fraction. We will spare the reader from this discussion.

\section{APPLICATION TO THE OBSERVED STATES AND THE STABILITY OF THE HIERARCHICAL JAIN STATES}

In this section we show that the states reported in Ref. [19], as well as some other states observed earlier on [20, 
40], can be reproduced within this framework.

It is clear from the experiments that all these states are not equally stable, and that some criterion must be devised to classify these states. We will address this problem here at a very qualitative (and perhaps naive) level.

In the case of the primary Jain sequences the stability of the states is determined by the size of the excitation gap and, to a lesser extent, by the interaction coupling constants between quasi-particles. Here too a similar sort of stability criterion can be constructed, by focusing mainly on the quasi-particle (and quasi-hole) energy gaps. We should note that an argument of this sort can at best determine the relative stability for states sharing the same parent Jain state. In particular, this sort of analysis should fail if a competing state of a different type were to be allowed for the same filling factor. Another factor that must be taken into account is that the states can be FQH states of either quasi-particles or quasi-holes, which in general are not related simply by a particle-hole transformation as they have different gaps as well as interactions, and particle-hole symmetry holds only approximately.

A related issue is that in our construction of the hierarchical states there is more than one way to reproduce a given filling fraction. For instance the $\nu=4 / 11$ state can be obtained as a $1 / 3$ descendant with $\nu_{1}=1 / 3$, or alternatively, as a $2 / 5$ descendant with $\nu_{1}=-2 / 3$. Likewise, the $\nu=5 / 13$ state can be constructed as a $1 / 3$ descendant with $\nu_{1}=2 / 3$, or as a $2 / 5$ descendant with $\nu_{1}=-1 / 3$. In the case of $\nu=4 / 11$ the choice seems straightforward. It should be a descendant of the more stable parent state $(1 / 3)$ with the smallest possible number of extra quasi-particles condensing into a $\nu_{1}=1 / 3$ state. However, for the $5 / 13$ state it is not clear whether the correct option is to choose the more stable parent state $(1 / 3)$ with a larger number of condensing quasiparticles into a $\nu_{1}=2 / 3$ state, or the less stable parent state $(2 / 5)$ but with a smaller condensate of quasi-holes into a $\nu_{1}=-1 / 3$. In the case of exact particle-hole symmetry these two constructions lead exactly to the same state even though they have different parent states. Notice that even though there are different ways to construct a given hierarchical state, the resulting state has exactly the same universal properties. Hence these are not distinct FQH states.

In what follows, we will discuss two different criteria which are both intuitively reasonable and are natural within the framework of this mean field theory. A more careful study of the energy gaps and of the interactions between the elementary excitations (which is beyond the scope of this work) is necessary to determine which of the following criteria is more suitable.

The first criterion is based on the following assumptions:

1. The more stable parent states are the Jain states with larger gaps.

2. The more stable hierarchical states are those that having the largest gaps, are descendants of the more stable Jain states.

3. If there are two different ways of constructing a hierarchical state, the more stable one is the one that has the more stable parent state, and that requires the lowest density of condensing quasi-particles.

4. Finally, states with $\nu_{1}>0$ are more stable than those with $\nu_{1}<0$, since quasi-holes are expected in general to have smaller excitations energies than quasi-particles.

It is apparent that this a rather qualitative criterion at best, and that in a number of cases it does not provide for a unique answer. For instance, it is not obvious whether it is better to construct a state with quasi-particles (with $\left.\nu_{1}>0\right)$ of a more stable primary state, or with quasiholes (with $\nu_{1}<0$ ) of a less stable primary state. Clearly it is not possible to determine a unique choice without some knowledge of the quasi-particle and quasi-hole excitation energies (their gaps) and of their interactions [41]. Nevertheless it is still useful to explore what consequences follow from the naive application of this criterion.

According to this criterion we must first look at the the states which share the same parent primary Jain state:

- $1 / 3$ descendants: they are obtained by setting $p=1$ and $2 n=2$, and are given by the sequences

$$
\frac{1}{\nu}=2+\frac{1}{1+\nu_{1}}
$$

yielding states at filling factors $4 / 11$ (with $\nu_{1}=$ $1 / 3$ ), $5 / 13$ (with $\nu_{1}=2 / 3$ ) and $6 / 17$ (with $\nu_{1}=$ $1 / 5)$. Other descendants, not seen yet in experiment, have filling factors $7 / 19\left(\nu_{1}=2 / 5\right), 10 / 27$ $\left(\nu_{1}=3 / 7\right), 8 / 21\left(\nu_{1}=3 / 5\right)$, and so on

- $2 / 3$ descendants: they are obtained by setting $p=$ -2 and $2 n=2$, and are given by the sequences

$$
\frac{1}{\nu}=2+\frac{1}{-2+\nu_{1}}
$$

According to our criterion, the most stable descendant states will be $5 / 7$ (with $\nu_{1}=1 / 3$ ), 9/13 (with $\nu_{1}=1 / 5$ ), 4/5 (with $\nu_{1}=2 / 3$ ), 8/11 (with $\left.\nu_{1}=2 / 5\right)$, etc. All these states fall outside of the range of filling fractions of the data of Ref. [19], since they have $\nu>2 / 3$. However, a weak signature of a possible state at $5 / 7$ and $4 / 5$ was observed earlier by Du et al. [40] who saw a weak depression of the longitudinal resistivity for these filling factors. Also, Goldman and Shayegan [20] saw FQH states at $\nu=9 / 13$ (and at $7 / 11)$.

- The $2 / 7$ descendants are obtained by setting $p=$ -2 and $2 n=4$, and are given by the sequences

$$
\frac{1}{\nu}=4+\frac{1}{-2+\nu_{1}}
$$


The states seen in the experiment have filling factors at $4 / 13$ (with $\nu_{1}=2 / 3$ ), and $5 / 17$ (with $\left.\nu_{1}=1 / 3\right)$.

- The $3 / 5$ descendants are obtained by setting $p=$ -3 and $2 n=2$, and are given by the sequences

$$
\frac{1}{\nu}=2+\frac{1}{-3+\nu_{1}}
$$

So-far the only observed $3 / 5$ descendant has filling factor $7 / 11$ (with $\nu_{1}=2 / 3$ ).

A somewhat different classification of the observed states can be obtained if we adopt another stability criterion. In this second criterion we will assume instead that the more stable states are those generated by the condensation of the smallest number of elementary excitations on top of the more stable Jain states, independently of whether they are quasi-particles or quasi-holes. This is a very crude approximation, but consistent with our derivation of the quasi-particle condensate, since except for the sign of the charge, it does not distinguish between quasi-particles and quasi-holes.

It is straightforward to show that given a parent state with filling fraction $1 /(2 n+1 / p)$, the number of elementary excitations on top of it that condense into the state $\nu=\frac{p\left(2 n_{1} p_{1}+1\right)+p_{1}}{|\operatorname{det} K|}$ is given by $n_{1}^{q p}=\frac{B}{2 \pi} \frac{p_{1}}{\operatorname{det} K \mid}$ (in units of $e=c=\hbar=1)$. Therefore, if there are two different ways of generating a given filling fraction, the more stable one will be the one that has the smallest number of condensing excitations, $i$. e., the smallest $p_{1}$. According to this criterion, for each Jain state, the more stable descendants, and therefore the most likely to be observed, will be those with $\nu_{1}= \pm 1 /\left(2 n_{1}+1\right)\left(i\right.$. e., $\left.p_{1}= \pm 1\right)$. We will see below that all the states generated following this rule have been seen in reference 19].

Thus, following this second criterion we get instead the following classification:

- $1 / 3$ descendants: In this case, the more stable descendant states will be $4 / 11$ (with $\nu_{1}=1 / 3$ ), 6/17 (with $\nu_{1}=1 / 5$ ), and $4 / 13$ (with $\left.\nu_{1}=-1 / 5\right)$. For $\nu_{1}=-1 / 3$ we obtain $\nu=2 / 7$ which is of course more stable as a Jain (parent) state. The next states that can be obtained are $8 / 23\left(\nu_{1}=1 / 7\right)$, and $6 / 19\left(\nu_{1}=-1 / 7\right)$, whose gaps are smaller than the ones observed by Pan et al. [19].

- $2 / 3$ descendants: This yields the states $5 / 7$ (with $\nu_{1}=1 / 3$ ), $7 / 11$ (with $\nu_{1}=-1 / 3$ ), 9/13 (with $\left.\nu_{1}=1 / 5\right), 11 / 17$ (with $\nu_{1}=-1 / 7$ ), etc. The state 9/13 lies outside the range of the data of Ref. 19] (since $9 / 13>2 / 3$ ), although it was reported earlier in Ref. 20. However, 11/17 is within the range of the observed states, and in principle should be as stable as $5 / 17$ or $6 / 17$, except for the fact that it is the result of a quasi-hole condensate.

- $2 / 5$ descendants: This yields the states $3 / 17$ (with $\left.\nu_{1}=1 / 3\right)$, and $5 / 13\left(\right.$ with $\left.\nu_{1}=-1 / 3\right)$. Notice that
$3 / 17$ being smaller than $2 / 7$ is out of the range of the states observed in 19]. The next possible states $5 / 27$ (with $\nu_{1}=1 / 5$ ), $9 / 23$ (with $\nu_{1}=-1 / 7$ ), etc, have very small gaps. The state $5 / 13$ can also be constructed as a $1 / 3$ descendant with $\nu_{1}=2 / 3$, but this choice requires a larger number of condensing excitations than the one obtained as a $2 / 5$ descendant.

- 2/7 descendants: have filling factors $5 / 17$ (with $\left.\nu_{1}=1 / 3\right), 7 / 25$ (with $\nu_{1}=-1 / 3$ ), and states with even smaller gaps.

- 3/5 descendants: have filling factors $8 / 13$ (with $\nu_{1}=1 / 3$ ), 10/17 (with $\nu_{1}=-1 / 3$ ), and states with even smaller gaps. As discussed before, the state $7 / 11$ can be constructed as a $3 / 5$ descendant with $\nu_{1}=2 / 3$, but this requires a larger number of quasi-particles than the construction as a $2 / 3$ descendant.

As mentioned before, besides the very general arguments invoked about the stability of the parent states, together with the size of the gap and the density of condensing excitations, there are no other factors that one can take into account at this level of approximation to decide on how to organize the states according to their degree of relative stability. A microscopic study of the interactions between the quasi-particles and/or quasi-holes involved is necessary to solve this question. Effective interactions among excitations of Jain states have been studied numerically recently by Lee et al. 42]. Goerbig et al. 43] have investigated the form of the quasi-particle interactions for Jain states with $\nu=\frac{1}{2 n+1}$ using the Hamiltonian approach for the fermionic Chern-Simons theory of the FQHE of Murthy and Shankar [14].

To conclude our discussion about the experimental results, we turn our attention to the minima in $\rho_{x x}$, but not yet a plateau in $\rho_{x y}$, observed by Pan et al. [19] at the even denominator fractions. We can construct these states as Jain descendants only if $\nu_{1}=1 / 2$. In particular, we obtain $3 / 8$ as a $1 / 3$ descendant, $3 / 10$ as a $2 / 5$ descendant, and $5 / 8$ as a $3 / 5$ descendant. However, in this theory these even-denominator states are compressible [37] fully polarized hierarchical Jain states since they are gapless. Alternatively, they have been described as either paired states 24 25 44], or liquid crystallike states 454647$]$.

\section{A PARTIALLY SPIN POLARIZED DESCRIPTION OF THE OBSERVED STATES}

Up to this point, we have discussed how to construct the FQHE states observed in Ref. [19] as fully polarized hierarchical descendants of the Jain series. Since this construction requires the condensation of the quasi-particles of the parent state, it is clear that these daughter states might become unstable under changes leading to a complete reorganization of the ground state. Thus, if a FQH 
state of electrons were to become available at the same filling fraction, for instance a state which involves the spin degrees of freedom, it will be necessary to determine which state is chosen for a given physical system. Thus, the application of pressure to the sample, or tilting of the magnetic field may drive a transition to a partially polarized, or even an unpolarized state. Similar considerations apply to paired states.

In this section we will consider if partially polarized FQH states can compete with the hierarchical Jain states discussed in Section [1] Ref. [18] and Ref. [48] we showed that the Chern-Simons field theory for two dimensional electron systems that have an extra degree of freedom, such as a layer or spin index, can describe FQHE states whose filling fractions are

$$
\nu=\frac{2 n-\left({\frac{1}{p_{\downarrow}}}+2 n_{\downarrow}\right)-\left({\frac{1}{p_{\uparrow}}}+2 n_{\uparrow}\right)}{n^{2}-\left({\frac{1}{p_{\uparrow}}}+2 n_{\uparrow}\right)\left({\frac{1}{p_{\downarrow}}}+2 n_{\downarrow}\right)}
$$

Here, $2 n_{\sigma}$ is the integer number of fluxes attached to the electrons with polarization $\sigma(\sigma=\uparrow, \downarrow)$, and $n$ is the integer number of fluxes attached to a given particle due to the presence of the particles with the opposite polarization. The integer $\left|p_{\sigma}\right|$ is the number of Landau levels filled by the particles with polarization $\sigma$. All these numbers can have either sign, since the flux attached, or the effective magnetic field seen at mean field, can be either parallel or anti-parallel to the external magnetic field.

The total $z$-component magnetization per electron of the ground state is

$$
S_{\text {total }}^{z}=\frac{\nu_{\uparrow}-\nu_{\downarrow}}{2 \nu}
$$

As discussed in Ref. [48] and Ref. [18], in the case of a spin- $1 / 2$ system, in order to preserve the $S U(2)$ spin rotation invariance of the Hamiltonian, even in the presence of a Zeeman term, we must choose $2 n_{\sigma}=n$. In other words, the flux attachment should be done in such a way that it does not distinguish between different spin orientations. In particular this guarantees that the statistics of all the quasi-particles is the same, independently of their spin orientation. Notice that this is a symmetry requirement and it does not imply that the state is necessarily a spin singlet (although it is consistent with it). It is simple to check that none of the states reported in Ref. [19] can be obtained imposing the condition $2 n_{\sigma}=n$, $\sigma=\uparrow, \downarrow$.

On the other hand, if we could assume that the physical system breaks the $S U(2)$ symmetry explicitly, we would be allowed to relax this condition and only require that the number of crossed flux $(n)$ attached to the particles is even in order to maintain their fermionic statistics. In this case it is possible to find realizations of the different observed states. Taking for instance $n=2 n_{\uparrow}$ and $2 n_{\downarrow}-$ $2 n_{\uparrow} \equiv 2 m$, we can rewrite the total filling fraction in the following way

$$
\nu=\frac{1}{2 n_{\uparrow}+\frac{1}{p_{\uparrow}+\nu^{*}}}
$$

where

$$
\nu^{*}=\frac{1}{2 m+\frac{1}{p_{\downarrow}}}
$$

This expression looks identical to the one we found for the hierarchical states (eq. (1.8) ) provided that $p_{\uparrow} \rightarrow p$, $2 m \rightarrow 2 n_{1}, p_{\downarrow} \rightarrow p_{1}$, and $2 n_{\uparrow} \rightarrow 2 n$. However the interpretation of the integers appearing here is different. In this case the flux attachment is performed on the electrons, with $2 n_{\uparrow}=2 n$ fluxes attached to electrons with spin $\uparrow$, and $2 n_{\downarrow}=2 n+2 n_{1}$ fluxes attached to electrons with spin $\downarrow$. It is therefore obvious that the $S U(2)$ symmetry is explicitly broken. Moreover, all these states are partially polarized with polarization $S_{\text {total }}^{z}=\frac{p_{\uparrow}\left(2 m p_{\downarrow}+1\right)-p_{\downarrow}}{2\left[p_{\uparrow}\left(2 m p_{\downarrow}+1\right)+p_{\downarrow}\right]}$. On the other hand, in the case of the hierarchical construction of Section [1 $2 n$ fluxes are attached to the original electrons that condense into the parent state, and $2 n_{1}$ fluxes to the remaining excitations that condense into the daughter state.

It can be shown that the states with even denominator $(\nu=3 / 8,5 / 8$ and $3 / 10)$ can not be constructed as incompressible partially polarized ones if the condition $n=2 n_{\uparrow}$ is imposed. We could only obtain them if $\nu^{*}=1 / 2 m$, but in this case, they are compressible. Other possible mechanisms to generate even denominator incompressible states involve pairing [24 25].

An alternative proposal has been put forth by Park and Jain [49], who discussed states whose filling fraction is given by Eq. (3.3) for the particular case of $n=2, p_{\uparrow}=1$. The authors argue that these states are mixed states of composite fermions of different flavors, carrying different number of fluxes (vortices). In fact, the construction of Ref. 49] can be obtained from a scheme similar to the partially polarized states discussed in this section. Thus, we first attach $n=2 n_{\uparrow}$ fluxes to every fermion, independently of the orientation of its spin. The remaining fluxes $2 n_{\downarrow}-2 n_{\uparrow}=2 m$ are attached only to the, let's say, spin $\downarrow$ electrons.

However, as discussed above, a construction of this type, in which different spin orientations get attached different number of flux quanta, breaks explicitly the $S U(2)$ spin symmetry of the system, and it is not an equivalent representation of the system. Naturally, it is always possible to carry out the formal process of flux attachment in an asymmetric fashion, $i$. $e$. by ignoring the fact that, except for the presence of the Zeeman term (which commutes with the Hamiltonian), the physical system is $S U(2)$ invariant. In such an approach, spin $S U(2)$ becomes a dynamical symmetry. However, as soon as approximations are made, the $S U(2)$ symmetry is explicitly broken. Of course, since the original problem has an $S U(2)$-symmetric Hamiltonian, the effects of the symmetry should eventually be recovered. However there is no guarantee that the symmetry can be recovered in perturbation theory, as it may well be a non-perturbative effect. Thus, the resulting mean field theory of composite fermions is constructed with states which are essentially orthogonal to the physical states, and the fully projected 
states are very far from the intuitive and simple wave functions of (composite) fermions filling up these effective asymmetric Landau levels. Thus, although Park and Jain succeeded in constructing states of this type for most of the new fractions, the states they find are not fully polarized. Moreover, it is unclear how this scheme can be made compatible with the spin symmetry of the underlying Hamiltonian. We should emphasize that, to have a ground state which is either fully or partially polarized, it is not necessary to break the $S U(2)$ symmetry of spin at the level of the Hamiltonian (beyond the effects of a possible Zeeman term).

\section{EDGE STATES AND ELECTRON TUNNELING}

In this Section we give a brief derivation of the theory of the edge states for the hierarchical Jain states discussed in Section II Since we will follow closely the approach we used in Ref. 17] and Ref. [18] we will omit many details and refer the reader to these references. The main goal of this section is to determine the exponent of the differential tunneling conductance for electrons.

The Lagrangian of Eq. (1.5) has the standard form discussed by Wen and Zee 6]. Therefore, following the general arguments of Ref. [5], it is straightforward to extract a theory for the edge states which reflects the structure of the bulk. In what follows we assume that there is a sharp potential that confines the electrons to a disk in such a way that there is no edge reconstruction. Furthermore we will also assume [17 18] that the possible multiple edge charge modes are unresolved, $i$. e. that they are confined within a magnetic length. Of course the validity of this assumption depends on microscopic details such as the specific form of the confining potential.

The effective theory takes its simplest form when written in terms of the chiral boson $\phi_{C}$, the charge mode, which is the only mode that couples to the electromagnetic field, and non-propagating topological modes which play a role similar to that of Klein factors. In terms of these fields, the Lagrangian for the edge theory is

$$
\begin{aligned}
\mathcal{L} & =\frac{1}{4 \pi \nu}\left(\partial_{1} \phi_{C} \partial_{0} \phi_{C}-v \partial_{1} \phi_{C} \partial_{1} \phi_{C}\right) \\
& -\frac{1}{4 \pi}\left(p+\nu_{1}\right) \partial_{1} \phi_{T} \partial_{0} \phi_{T} \\
& -\frac{1}{4 \pi} \kappa_{i j} \partial_{1} \phi_{T_{i}} \partial_{0} \phi_{T_{j}}
\end{aligned}
$$

where $v$ the velocity of the charged edge mode. The $2 \times 2$ matrix $\kappa_{i j}$ is given by

$$
\kappa=\left(\begin{array}{cc}
p & 1 \\
1 & -2 n_{1}
\end{array}\right)
$$

A general edge operator can be written as

$$
\Psi(x)=e^{i\left(\alpha_{C} \phi_{C}+\alpha_{T} \phi_{T}+\sum_{i=1}^{2} \alpha_{T_{i}} \phi_{T_{i}}\right)}
$$

The charge $Q$ and statistics $\theta$ of these excitations are

$$
\begin{aligned}
& \frac{Q}{e}=-\nu \alpha_{C} \\
& \frac{\theta}{\pi}=-\nu \alpha_{C}^{2}+\frac{1}{p+\nu_{1}} \alpha_{T}^{2}+\alpha_{T_{i}} \kappa_{i j}^{-1} \alpha_{T_{j}}
\end{aligned}
$$

It is apparent that the topological modes only contribute to determine the statistics of the operators. Their role is to provide for a set of effective Klein factors which give the physical excitations their correct statistics.

The operator that creates a quasi-particle or quasihole at the boundary can be found by requiring that its charge and statistics are given by Eqs. (1.11) and (1.12) respectively. It is immediate to see that the quasi-hole operator can be written as

$$
\Psi_{\mathrm{qh}}(x)=e^{i\left(\frac{1}{p\left(2 n_{1} p_{1}+1\right)+p_{1}} \phi_{C}+\phi_{T}\right)}
$$

Analogously, the electron operator at the boundary is

$$
\Psi_{\mathrm{e}}(x)=e^{i\left(\frac{1}{\nu} \phi_{C}+|\operatorname{det} K| \phi_{T}\right)}
$$

This operator has the correct charge $Q=-e$, and statis$\operatorname{tics} \theta / \pi=|\operatorname{det} K|\left(2 n_{2}(2 n p+1)+2 n\right)$, measured with respect to fermions. Notice that all the physical operators can be represented only in terms of the charge mode $\phi_{C}$ and one of the topological modes $\phi_{T}$.

There is much interest in studying different tunneling processes into the edges of the FQHE hierarchical states. In order to do so, we need to compute the propagator for an excitation created by an operator of the form of Eq. (4.3). Since the effective action for the edge modes is quadratic in the fields, the calculation of the propagators of the chiral bosons is straightforward giving [17 18$]$

$$
\begin{aligned}
& <\phi_{C}(x, t) \phi_{C}(0,0)>=-\nu \ln z \\
& <\phi_{T}(x, t) \phi_{T}(0,0)>=i \frac{\pi}{2\left(p+\nu_{1}\right)} \operatorname{sgn}(x t)
\end{aligned}
$$

where $z=x+i v t$.

Using the above results, the propagator for the field $\Psi(x)$, of the form given in Eq. 4.3), in the limit $x \rightarrow 0^{+}$ becomes

$$
<\Psi^{\dagger}\left(0^{+}, t\right) \Psi(0,0)>\propto \frac{1}{|t|^{g_{t}}} e^{i \frac{\theta}{2} \operatorname{sgn}(t)}
$$

where $g_{t}=\nu \alpha_{C}^{2}$ and $\theta$ is given by Eq. 4.4. In particular, we find that the exponent $g_{t}$ for electrons is $g_{e}=1 / \nu$, whereas for quasi-particles (and quasi-holes) $g_{\mathrm{qp}}=\nu /\left(p\left(2 n_{1} p_{1}+1\right)+p_{1}\right)^{2}$.

The tunneling current $I$ at bias voltage $V$ has the scaling form [33 34] $I(V) \propto V^{\alpha}$, where the exponent $\alpha$ is determined by the scaling dimension of the tunneling operator. There are three cases of physical interest: (a) internal tunneling of quasi-particles, for which $\alpha_{q p}=2 g_{q p}-1$ (here scaling holds at large bias $V$ ), (b) tunneling of electrons between identical fluids, for which $\alpha_{e}=2 g_{e}-1$ (at low bias $V$ ), and (c) electron tunneling between distinct 
fluids, for which $\alpha_{t}=g_{e}$ (again at low bias $V$ ). In particular, in the case of tunneling of electrons from a Fermi liquid into a hierarchical FQH state, we find that the tunneling exponent is $\alpha=g_{e}=1 / \nu$.

Thus, in contrast with what is obtained for the Haldane-Halperin hierarchy [5], all the states described by our approach have a universal value of their electron tunneling exponent, which is always equal to $1 / \nu$ in the case of an unreconstructed unresolved edge. Although this interesting result agrees with the experimental observations of references 28 29 30 31], we should note that it does not predict that the exponent is $1 / \nu$ for all values of the magnetic field, as the experiments seem to suggest. Instead what this theory predicts is that for each FQH state at filling factor $\nu$, the exponent will lock at the value $1 / \nu$ so far as the bulk state has not changed. Thus, there should still be a plateau, no matter how small, centered about the value $1 / \nu$. Although this reproduces the trend seen in the experiment, it still requires that the functional dependence of the exponent on the magnetic field has the same structure as the Hall conductance. The fact that, with the possible exception of a very small plateau at $1 / 3$ with a small but observable discrepancy in the value of the exponent 29], no plateau has ever been seen in edge tunneling exponent remains an unsolved and intriguing puzzle which presumably will not be resolved until a true point-contact geometry becomes available for experiments.

\section{CONCLUSIONS}

In this paper, we presented an effective theory to describe hierarchical states constructed as daughter states of the principal fractional quantum Hall Jain series at $\nu=\frac{p}{2 n p+1}$. These daughter states result from the con- densation of the elementary excitations (either quasiparticles or quasi-holes) of the parent (Jain) state, into new FQH Jain-like states. This effective theory is a generalization of the fermionic Chern-Simons theory of the FQHE for fully polarized systems 9 17 18]. It reproduces all the odd denominator filling fractions reported in Ref. [19] as well as some states reported earlier in Refs. [20.40]. We found that these states are both locally stable and $T$-stable.

Our results do not preclude the existence of other possible competing states, such as paired states, inhomogeneous states (stripes, bubbles, etc.) or anisotropic nematic states. But they do confirm that at least locally (in energy space) the hierarchical states are stable states of the 2DEG.

We also used a similar approach to discuss the possibility of explaining some of these states as partially polarized FQH states. We found that it is not possible to construct the new states reported in Ref. [19] using a scheme which respects the $S U(2)$ invariance of the underlying Hamiltonian.

Finally, we derived a theory of the edge states for the new hierarchical states, using the Chern-Simons bulk effective theory presented here. In particular we discussed the predictions of this theory for the edge tunneling exponent of electrons from a Fermi liquid, and find that for an unreconstructed sharp unresolved edge, the tunneling exponent is always equal to $1 / \nu$ for all the fully polarized Jain states (hierarchical or not).

\section{Acknowledgments}

We thank W. Pan for useful discussions. This work was supported in part by the NSF grant DMR 01-32990 $(\mathrm{EF})$, and by CONICET, Argentina (AL).
* Permanent address: Centro Atómico Bariloche, (8400) S. C. de Bariloche, Río Negro, Argentina

1 R. B. Laughlin, Phys. Rev. Lett. 50, 1395 (1983).

2 B. I. Halperin, Phys. Rev. Lett. 52, 1583 (1984).

3 F. D. M. Haldane, Phys. Rev. Lett. 51, 605 (1983).

4 S. C. Zhang, T. H. Hansson, and S. A. Kivelson, Phys. Rev. Lett. 62, 82 (1989).

5 X. G. Wen, Adv. Phys. 44, 405 (1995).

${ }^{6}$ X. G. Wen and A. Zee, Phys. Rev. B 46, 2290 (1992).

7 S. A. Kivelson, D. H. Lee, and S. C. Zhang, Phys. Rev. B 46, 223 (1992).

8 J. K. Jain, Phys. Rev. Lett. 63, 199 (1989).

9 A. López and E. Fradkin, Phys. Rev. B 44, 5246 (1991).

10 P. Sitko, K.-S. Yi, , and J. J. Quinn, Phys. Rev. B 56 , 12417 (1997).

11 R. G. Mani and K. von Klitzing, Z. Phys. B 100, 635 (1996), constructed the hierarchical Jain fractions by means of a set of formal operations (reflections and translations) on the curve of the quantum Hall conductance for the integer QH effect.
12 R. Shankar and G. Murthy, Phys. Rev. Lett. 79, 4437 (1997).

13 G. Murthy and R. Shankar, Phys. Rev. B 65, 245309 (2002).

14 G. Murhty and R. Shankar (2002), cond-mat/0205326; to appear in Reviews of Modern Physics, October 2003.

15 A. Wójs and J. J. Quinn, Phys. Rev. B 61, 2846 (1999).

16 F. D. M. Haldane, Phys. Rev. Lett. 74, 2090 (1995).

17 A. López and E. Fradkin, Phys. Rev. B 59, 15323 (1999).

18 A. López and E. Fradkin, Phys. Rev. B 63, 085306 (2001).

19 W. Pan, H. Stormer, D.C.Tsui, L. Pfeiffer, K. Baldwin, and K. West, Phys. Rev. Lett. 90, 016801 (2003).

${ }^{20}$ V. J. Goldman and M. Shayegan, Surf. Science 229, 10 (1990).

21 S. S. Mandal and J. K. Jain, Phys. Rev. B 66, 155302 (2002).

22 C.-C. Chang, S. S. Mandal, and J. K. Jain, Phys. Rev. B 67, 121305 (2003).

23 Current state-of-the-art finite size numerical diagonalizations are done on clusters with up to 24 electrons in the 
lowest Landau level. For such large clusters, finite size effects should be small for filling factors in the primary Laughlin and Jain states. However, for hierarchical states a large fraction of the electrons are effectively "used up" in making up the underlying primary FQH state, and relatively few electrons (about seven) are left to make quasiparticles which should eventually condense. Thus, hierarchical states are expected to have significantly larger finite size effects compared to their parent primary states.

${ }^{24}$ V. W. Scarola, J. K. Jain, and E. H. Rezayi, Phys. Rev. Lett. 88, 216804 (2002).

25 A. Wójs, K. S. Yi, and J. Quinn (2003), condmat/0304130.

26 J. J. Quinn, A. Wójs, and K.-S. Yi (2003), condmat/0309435.

27 It is quite likely that there are phase transitions between these inequivalent states with the same filling fraction controlled by a fine tuning of microscopic parameters, e. $g$. the areal density of the 2DEG. For instance it is possible to have a phase transition between a fully polarized hierarchical FQH state at $\nu=4 / 11$ and the paired state also at 4/11 proposed in Ref. [26]. If true this would be a most interesting phase transition between two FQH states which differ only in their internal topological structure.

28 A. M. Chang, L. N. Pfeiffer, and K. W. West, Phys. Rev. Lett. 77, 2538 (1996).

29 M. Grayson, D.C.Tsui, L. Pfeiffer, K. West, and A. M. Chang, Phys. Rev. Lett. 80, 1062 (1998).

30 M. Hilke, D. C. Tsui, M. Grayson, L. N. Pfeiffer, and K. W. West, Phys. Rev. Lett. 87, 186806 (2001).

${ }^{31}$ M. Hilke, D. C. Tsui, L. N. Pfeiffer, and K. W. West, J. Phys. Soc. Jpn. 72, 92 (2003).

32 Naturally, this result means that the exponent has a plateau in the value of the exponent, no matter how small, whenever the bulk is in a FQH state with a given Hall conductivity.

33 X. G. Wen, Phys. Rev. B 44, 5708 (1991).

34 C. Kane and M. P. A. Fisher, Phys. Rev. Lett. 68, 1220 (1992).

35 C. Kane, M. P. A. Fisher, and J. Polchinski, Phys. Rev. Lett. 72, 4129 (1994).

36 A. V. Shytov, L. S. Levitov, and B. I. Halperin, Phys. Rev. Lett. 80, 141 (1998).

37 B. I. Halperin, P. A. Lee, and N. Read, Phys. Rev. B 47, 7312 (1993).
38 Estimates of these interactions for just two quasi-particles above the $\nu=1 / 3$ Laughlin state have been calculated for small clusters of electrons, and thus with presumably significant finite-size effects, in Refs. 25 26 50]. These results suggest that the gap is significantly smaller than the mean field estimate of Eq. (1.10), and that in certain models it may even vanish. In fact, it has been suggested that the new FQH state at $\nu=4 / 11$ is either partially polarized 2122 ] or paired 25 26].

39 The state at $\nu=4 / 11$ appears already at the first level of the Halperin-Haldane hierarchy where it results from the condensation of multiple vortices (or quasiholes) of the parent $1 / 3$ state. Using the notation of Refs. [2 3 5], it is constructed as the state with $p_{1}=3$ and $p_{2}=4$. In this sense it is less stable than the $2 / 5$ state which involves the condensation of quasiholes of the parent $1 / 3$ state, and has $p_{1}=3$ but $p_{2}=2$. Notice that in both cases the $K$-matrix is $2 \times 2$ and does not have null vectors. Thus, contrary from what a cursory reading of Ref. [16] may suggest, the state at $4 / 11$ is $T$-stable.

40 R. R. Du, H. L. Störmer, D. C. Tsui, A. S. Yeh, L. N. Pfeiffer, and K. W. West, Phys. Rev. Lett. 73, 3274 (1994).

41 Although in most situations the quasi-hole gap is expected (for the same state) to have a smaller gap than a quasiparticle, there are model interactions for which the order may be reversed. However this does not appear to be the case in any of the published studies. See, for instance, Refs. [42 43].

42 S.-Y. Lee, V. W. Scarola, and J. Jain, Phys. Rev. B 66, 085336 (2002).

43 M. O. Goerbig, P. Lederer, and C. Morais Smith, Phys. Rev. B 69, 155324 (2004), cond-mat/0308021.

44 N. Read and G. Moore, Nucl. Phys. B 360, 362 (1991).

45 S. Y. Lee, V. W. Scarola, and J. K. Jain, Phys. Rev. Lett. 87, 256803 (2001).

46 A. A. Koulakov, M. M. Fogler, and B. I. Shklovskii, Phys. Rev. Lett. 76, 499 (1996).

47 R. Moessner and J. T. Chalker, Phys. Rev. B 54, 5006 (1996).

48 A. López and E. Fradkin, Phys. Rev. B 51, 4347 (1995).

49 K. Park and J. K. Jain, Phys. Rev. B 62, R13274 (2000).

50 A. Wójs, J. J. Quinn, and L. Jackak (2003), condmat/0308409. 